\title{
Highlights from Transcatheter Cardiovascular Therapeutics 2009: San Francisco, California, September 21 to 25, 2009
}

\author{
Jeffrey M. Sparling, MD, and Frederic S. Resnic, MD, MSc, FACC
}

Transcatheter Cardiovascular Therapeutics, the annual interventional cardiology scientific symposium, was held September 21 to 25, 2009, in San Francisco, California. This internationally attended meeting was the venue for the release of new clinical trial data and an ongoing comprehensive review of many subjects of interest to the cardiothoracic surgical community.

\section{OUTCOMES IN 3-VESSEL CORONARY ARTERY DISEASE AND LEFT MAIN CORONARY ARTERY DISEASE AFTER REVASCULARIZATION}

The SYNTAX investigators presented an ongoing analysis examining outcomes in patients requiring repeat revascularization after initial coronary artery bypass grafting (CABG) or multivessel or left main (LM) percutaneous coronary intervention (PCI). Of the 1800 patients initially enrolled, 224 required a repeat procedure, primarily in the first 6 months after the index revascularization. The primary need for a second procedure was recurrent angina, regardless of arm of enrollment. Patients undergoing CABG as the initial procedure required less repeat revascularization than those receiving multivessel stents $(8.6 \%$ vs $17.4 \%$, $P<.001)$. Predictors of the need for a repeat revascularization included medically treated diabetes mellitus in the PCI group and initial treatment for unstable angina or urgent revascularization in the CABG arm. Regardless of the type of initial treatment arm, "complete" revascularization at the time of index procedure reduced the need for a subsequent repeat revascularization. ${ }^{1}$

Investigators continue to examine the SYNTAX score as a means of predicting outcomes after percutaneous intervention in traditionally high-risk anatomy. PCI of unprotected (LM) coronary artery lesions seems to be as efficacious as CABG at 15 months for isolated LM disease when the SYNTAX score is acceptably low (indicating freedom from additional complex coronary artery disease). Dr Marie-Claude Morice presented data regarding contemporary indications for LM revascularization techniques in the trial. This was included in an analysis of the SYNTAX-LE MANS substudy,

\footnotetext{
From the Division of Cardiology, Brigham and Women's Hospital, Boston, Mass. Address for reprints: Frederic S. Resnic, MD, MSc, FACC, Division of Cardiology, 75 Francis St, Tower 3B, Brigham and Women's Hospital, Boston, MA 02115 (E-mail: fresnic@partners.org).

J Thorac Cardiovasc Surg 2010;139:515-7

$0022-5223 / \$ 36.00$

Copyright (c) 2010 by The American Association for Thoracic Surgery

doi:10.1016/j.jtcvs.2009.12.013
}

which was designed to compare the angiographic characteristics and symptoms of restenosis in large epicardial vessels, such as the LM coronary artery in the SYNTAX trial. The 15-month angiographic evaluation of this subset of patients revealed that $92 \%$ of $\mathrm{LM}$ drug-eluting stents were still widely patent (particularly in those patients with a low SYNTAX score) and that $26 \%$ of patients who underwent CABG had at least 1 graft totally occluded, although graft occlusion had no clinical consequence in terms of major adverse clinical events. ${ }^{2}$

A 4-year review examining the predictive value of the SYNTAX score in LM PCI outcomes studied consecutive patients undergoing CABG or PCI for isolated LM disease or LM disease with associated 1-vessel coronary artery disease. Results were similar to those reported in SYNTAX, reflecting that mortality trends in PCI directly correlated with SYNTAX score. An increased score directly correlated with increased mortality in patients undergoing LM PCI compared with CABG, whereas outcomes after PCI in patients with low scores were similar to those after surgical revascularization. ${ }^{3}$

A similar study from Italy comparing 5-year outcomes in unprotected LM PCI with CABG examined major adverse cardiac and cerebrovascular events in consecutive patients undergoing PCI or CABG. Investigators performed a propensity analysis examining outcomes 5 years after revascularization, illustrating that major adverse cardiac and cerebrovascular events were improved in the PCI cohort (11.2\% vs $20.4 \%, P=.004)$, whereas the rate of target vessel revascularization procedures was lower in the CABG group (28\% vs $8.4 \%, P=.0004){ }^{4}$

The accumulating evidence presented at Transcatheter Cardiovascular Therapeutics indicates that anatomic complexity, rather than LM coronary artery location by itself, predicts major clinical events after PCI, although longerterm follow-up of all studies is required.

\section{PHARMACOLOGY OF ACUTE CORONARY SYNDROMES AND CORONARY ARTERY DISEASE}

Multiple presentations were made on various pharmacologic agents used in the treatment of acute coronary syndrome (ACS), many of which have direct implications on the patient who has already undergone cardiac surgery or for whom CABG may be contemplated.

Deepak Bhatt, MD, presented the initial results of the COGENT trial, the first randomized clinical data regarding the interactions between clopidogrel and the proton pump inhibitor omeprazole. Previous observational studies, 
including a large cohort of more than 8200 patients examined in a retrospective manner over a 3 -year period, had suggested a possible negative interaction between the 2 classes of medication resulting in reduced efficacy of platelet inhibition, a clinically important scenario because many patients with cardiovascular disease who are receiving dual-antiplatelet therapy are receiving concomitant proton pump inhibitor therapy. ${ }^{5}$ The results strongly demonstrated a lack of interaction between the 2 medications as demonstrated by equivalent survival and rates of major adverse cardiac events, suggesting no reduction in clinical efficacy of clopidogrel in patients receiving omeprazole. Gastrointestinal end points were significantly increased in the group receiving clopidogrel and placebo compared with those receiving clopidogrel and omeprazole. ${ }^{6}$

Shamir Mehta, MD, presented results of the CURRENTSTEMI PCI study on behalf of the CURRENT investigators. This study was designed to examine the effects of doubledose clopidogrel $(600 \mathrm{mg}$ load, $150 \mathrm{mg}$ daily for 6 days, then $75 \mathrm{mg}$ daily ongoing) compared with standard clopidogrel dosing (300 $\mathrm{mg}$ load, then $75 \mathrm{mg}$ ongoing) in patients undergoing primary PCI for ST-elevation myocardial infarction (STEMI). This strategy had already been validated in CURRENT-OASIS 7 as significantly reducing ischemic events and stent thrombosis rates in patients with ACS who are undergoing percutaneous intervention. ${ }^{7}$ Of the 6346 patients with STEMI who were enrolled, the doubledose clopidogrel strategy significantly reduced ischemic events, including a $46 \%$ relative risk reduction in stent thrombosis, without increasing the risk of bleeding. ${ }^{8}$

The PLATelet inhibition and patient Outcomes trial sought to compare the novel oral, reversible P2Y12 platelet antagonist ticagrelor with clopidogrel in patients undergoing PCI for ACS or STEMI. Ticagrelor is unique because it is not a pro-drug (as clopidogrel is) and does not require metabolic activation, resulting in more rapid onset of platelet inhibition. Also, because it is reversibly bound it has a faster offset of action resulting in functional recovery of circulating platelets in approximately 48 hours, which has important implications for patients potentially needing both cardiac and noncardiac surgery while receiving dual-antiplatelet therapy. Chris Cannon, MD, on behalf of the PLATelet inhibition and patient Outcomes investigators, reported that in patients undergoing PCI, ticagrelor effectively reduced cardiovascular death, myocardial infarction, or stroke compared with clopidogrel $(9.02 \%$ vs $10.65 \%, P=.0025)$, without evidence of increased risk of bleeding. ${ }^{9}$

\section{SURGICAL TECHNIQUES AND OUTCOMES}

Additional pertinent topics during the week-long conference included reviews of cardiac and vascular surgery in elderly patients and diabetic patients; both advocated careful patient selection and evaluation when determining the optimal method of revascularization in these subgroups. In diabetic patients, patient profiling using operative risk scores such as EUROSCORE and SYNTAX can be useful tools to orient the clinician as to the optimal revascularization strategy, because diabetic patients with low-risk profiles can be adequately and effectively revascularized by both percutaneous and surgical means. ${ }^{10,11}$

Hybrid interventional and surgical coronary revascularization was the topic of significant discussion during the Transcatheter Cardiovascular Therapeutics meeting. The cost-effectiveness of hybrid revascularization was explored and reported demonstrative advantage for hybrid revascularization, particularly in the setting of high-risk patients with high SYNTAX scores. ${ }^{12}$ Many questions remain about the proper sequencing of a hybrid procedure (surgery first, PCI first, or simultaneous procedure), particularly as it relates to antithrombotic and antiplatelet strategies, and multiple presentations were made addressing this issue. Robert Poston, MD, presented single-center consecutive patient observational data of 55 patients undergoing hybrid CABG demonstrating that regardless of the order of the procedure, hybrid bypass/PCI was safe and effective. There was no difference in bleeding outcomes regardless of the sequence as measured by chest tube output and TIMI major bleeding score, even in the simultaneous procedures or the PCI-first procedures, for which all patients received preprocedural clopidogrel loading doses. ${ }^{13}$

Roxana Mehran, MD, presented a review on left internal thoracic artery (LITA) angiography after CABG. She reviewed early angiographic patency rates of LITA grafting postsurgically, including both traditional CABG and minimally invasive direct CABG. Review of data confirmed that the LITA remains an excellent conduit for bypass, with patency rates of approximately $95 \%$. She cautioned that clinical and angiographic variables should be carefully considered before the decision is made to intervene percutaneously on a LITA graft, especially in the early postoperative period, because the angiographic appearance of a LITA graft (especially at the anastomotic site) may vary depending on the healing process and flow characteristics. ${ }^{14}$

Advanced surgical techniques were presented in a main session on Wednesday, September 23. Michael Argenziano, $\mathrm{MD}$, discussed minimally invasive mitral valve surgery, including attempts to improve on the highly efficacious gold standard open valvular repair by improving the morbidity associated with the procedure (recovery time, wound complications, bleeding, and cosmesis) via the use of minimally invasive techniques. He presented the Columbia Hospital experience, including a propensity analysis of 1005 isolated mitral valvular repair cases performed via the standard or minimally invasive approach with 4-year follow up. Although aortic crossclamp time and cardiopulmonary bypass times were slightly increased in the minimally invasive group, length of stay and 30-day and 1-year mortality were decreased. ${ }^{15}$ 
Advances in surgically implanted ventricular assist devices were also presented, particularly focusing on the development and availability of future technologies. Robert Dowling, MD, discussed extensively current and future generations of left ventricular assist devices, including continuous-flow, chronic counterpulsation, and centrifugal pump devices that are either currently involved in or have just completed clinical trial assessment. Technology in this field continues to evolve toward smaller devices requiring lessinvasive surgical techniques for placement and reduction in the neurologic complications associated with placement and the need for associated anticoagulation. These developments all indicate a potential candidacy for ventricular assist device insertion in patients classically considered too "well" to undergo placement of a mechanical support device, which is precisely the patient population currently being randomized in the Randomized Evaluation of VAD InterVEntion before Inotropic Therapy trial, which Dr Dowling discussed. This National Institutes of Health-funded trial examines survival and quality of life indicators in patients with a ventricular assist device implanted who, before implant, were neither inotrope-dependent nor exercise-intolerant and who had no evidence of end-organ involvement. ${ }^{16}$

\section{References}

1. Feldman TE. Analysis of outcomes after repeat revascularization: analysis from the SYNTAX trial. Transcatheter Cardiovascular Therapeutics 2009, September 22, 2009, San Francisco, California.

2. Morice M. Contemporary indications for left main stenting: new insights from SYNTAX. Transcatheter Cardiovascular Therapeutics 2009, September 23, 2009, San Francisco, California.
3. Nadra I. Does the SYNTAX score predict outcome following PCI to the left main coronary artery in the real world? Transcatheter Cardiovascular Therapeutics 2009, September 22, 2009, San Francisco, California.

4. Chieffo A. Five year outcomes following PCI with DESimplantation vs CABG for unprotected LMCA lesions: Milan experience. Transcatheter Cardiovascular Therapeutics 2009, September 22, 2009, San Francisco, California.

5. Ho PM, Maddox TM, Wang L, et al. Risk of adverse outcomes associated with concomitant use of clopidogrel and proton pump inhibitors following acute coronary syndrome. JAMA. 2009;301:93.

6. Bhatt D. The COGENT trial. Transcatheter Cardiovascular Therapeutics 2009, September 23, 2009, San Francisco, California.

7. Mehta S.CURRENT OASIS 7: A $2 \times 2$ factorial randomized trial of optimal clopidogrel and aspirin dosing in patients with ACS undergoing an early invasive strategy with intent for PCI. European Society of Cardiology 2009 Scientific Sessions, August 30, 2009, Barcelona, Spain.

8. Mehta S.CURRENT-STEMI PCI: doubledose vs standard dose clopidogrel in ACS patients undergoing PCI for STEMI. Transcatheter Cardiovascular Therapeutics 2009, September 24, 2009, San Francisco, California.

9. Cannon C. PLATO-Invasive: ticagrelor compared with clopidogrel in patients with acute coronary syndromes-the Platelet Inhibition and Patient Outcomes Trial. Transcatheter Cardiovascular Therapeutics 2009, September 24, 2009, San Francisco, California.

10. Kappetein AP. Coronary revascularization in diabetic patients. Transcatheter Cardiovascular Therapeutics 2009, September 25, 2009, San Francisco, California.

11. Wimmer-Greinecker G. Coronary revascularization in elderly patients. Transcatheter Cardiovascular Therapeutics 2009, September 25, 2009, San Francisco, California.

12. Byrne JG. Hybrid revascularization: can it be cost effective? Transcatheter Cardiovascular Therapeutics 2009, September 24, 2009, San Francisco, California.

13. Poston R. Hybrid revascularization: simultaneous vs staged early vs staged delayed. Transcatheter Cardiovascular Therapeutics 2009, September 24, 2009 , San Francisco, California.

14. Mehran R. IMA angiography soon after CABG: findings and when to intervene Transcatheter Cardiovascular Therapeutics 2009, September 24, 2009, San Francisco, California.

15. Argenziano M. Minimally invasive mitral valve surgery: transferring the pain from the patient to the surgeon. Transcatheter Cardiovascular Therapeutics 2009, September 23, 2009, San Francisco, California.

16. Dowling RD. Emerging LVAD technologies. Transcatheter Cardiovascular Therapeutics 2009, September 23, 2009, San Francisco, California. 